\title{
New Generation of 3D Body Scanning Technologies New Possibilities for Fashion and Marketing
}

\author{
Jean-Loup RENNESSON \\ TELMAT Industrie SA, Soultz, France
}

\begin{abstract}
An analysis of the requirements for the 3D scanning of human bodies is given, including the following topics: time of scanning, calibration, 3D resolution, automatic detection of anatomical landmarks. In the framework of this analysis, the impact of the 3D scanning time on the accuracy of scan data and body measurements is studied.

Furthermore, we make an overview of the state of the art in 3D body scanning technologies, including technologies based on laser scanning, and those based on the projection of single-pattern structured light and on the projection of multiple-pattern structured light.

Then we describe the new generation of technologies, based on stereovision and on the projection of highly-encoded structured light (registered under the trademark SYMCAD ${ }^{\mathrm{TM}}$ ) and how the robustness of the 3D reconstruction and the 3D resolution may be enhanced by applying specific coding processes such as Gray encoding and phase shifting.

We assess the adequacy of this new technology for its use to scan living subjects because of its following features: 3D digitizing process within 0.5 seconds - automatic self-calibration within 5 seconds - automatic body measurement extraction - Special Tracking of active landmarks - creation of 3D avatars.

At last, we show the new possibilities offered by this new technology in fashion and marketing, as well as for other domains like ergonomics, health and entertainment.
\end{abstract}

Keywords: 3D body scanning technologies, automated body measurement, made-to-measure, mass customization

\section{Introduction}

The use of 3D body scanning systems is now common for the issue of military uniforms or corporate apparel. However its deployment in the fashion industry implies specific requirements such as easeof-use, consistency and reliability. This paper gives the details of these requirements for the 3D scanning of human bodies including the following topics: time of scanning, calibration process, resolution of captured 3D data, detection of anatomical landmarks and body measurement extraction. An overview of the state of the art and then the new generation of the SYMCAD ${ }^{\text {TM }}$ 3D body scanning technology are presented. The results achieved by this new technology are described into details and application areas are overviewed such as fashion and marketing, ergonomics, health and entertainment.

\section{State of the art of the 3D Body Scanning technologies}

\subsection{D Body Scanning technologies based on laser scanning}

The 3D scanning process is obtained by displacement of a set of emitters/sensors such as laser beams and moving CCD video cameras (see example in figure 1). Each point of 3-dimensional surfaces is obtained by triangulation of each point of a laser beam projected onto the object and viewed by the video camera (see figure 2).

The process of triangulation consists in determining the location of a 3D point of the object by measuring angles to it from known points (the projection centres of the video camera and the laser emitter). 
Depending on the distance at which the laser strikes the surface of the object from a reference point $(\Delta \mathrm{Z})$, the laser dot appears at different places in the camera's field of view $(\Delta \mathrm{z})$. This technique is called triangulation because the laser dot, the camera and the laser emitter form a triangle. The length of one side of the triangle, the distance between the camera and the laser emitter is known. The angle of the laser emitter corner is also known. The angle of the camera corner can be determined by looking at the location of the laser dot in the camera's field of view. This information gives the location of the laser dot corner of the triangle. In most cases a laser stripe, instead of a single laser dot, is swept across the object to speed up the acquisition process.

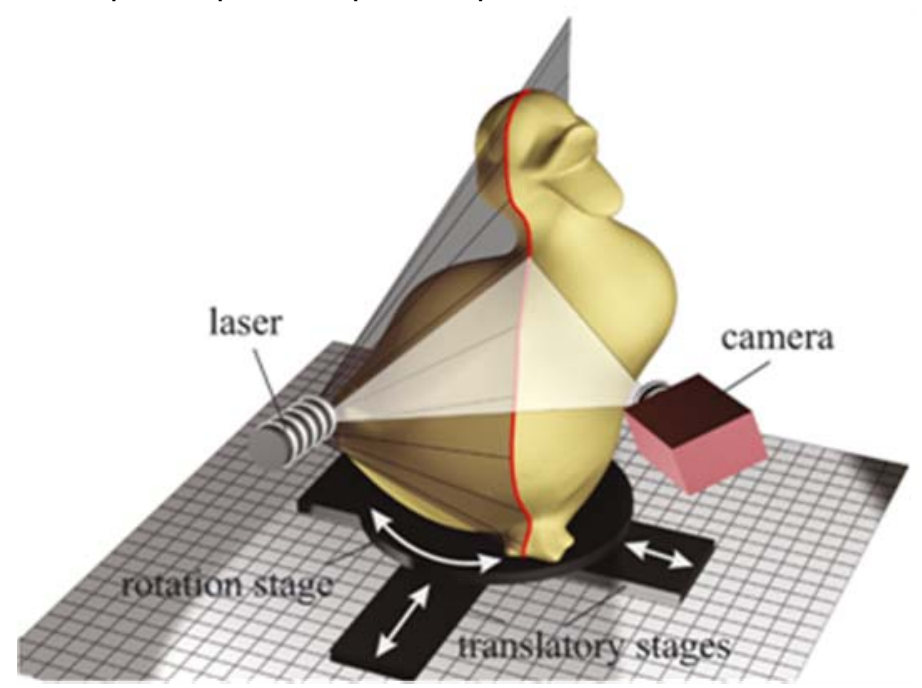

Fig. 1. Scanning of a static object (duck statue on a rotating plate) by a couple laser/camera

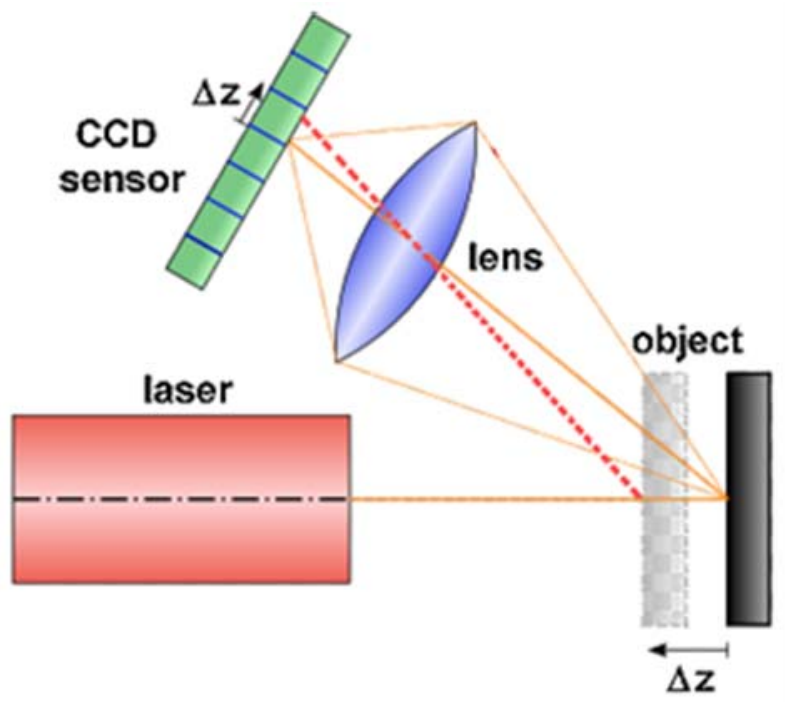

Fig. 2. Principle of triangulation by a couple laser/camera (CCD sensor)

The quality of 3-dimensional data reconstruction highly depends on the accuracy of the determination of optical centre of the video camera. Furthermore if the laser beam is not always projected through a fixed angle, the accuracy of the laser orientation must also be taken into account to determine the precision of the 3D laser scanner.

The 3-dimensional resolution depends on the time of scanning (vertical resolution) and on the CCD resolution (horizontal resolution). The time of scanning of a human subject takes from 12 to 24 seconds, depending on the vertical resolution to be achieved. The average resolution achieved by the laser 3D body scanners available on the market is comprised between 7 and 27 points $/ \mathrm{cm}^{2}$. 


\subsection{D Body Scanning technologies based on white light projection}

These technologies use no laser but a projector of structured light (white light i.e. not coherent light). The main benefit is the capacity of such technologies to reduce the total time of scanning because a full set of stripes are simultaneously projected onto the subject. The reconstruction of 3-dimensional surfaces is also based on triangulation between a video camera (CCD sensor) and a video projector (see figure 3).

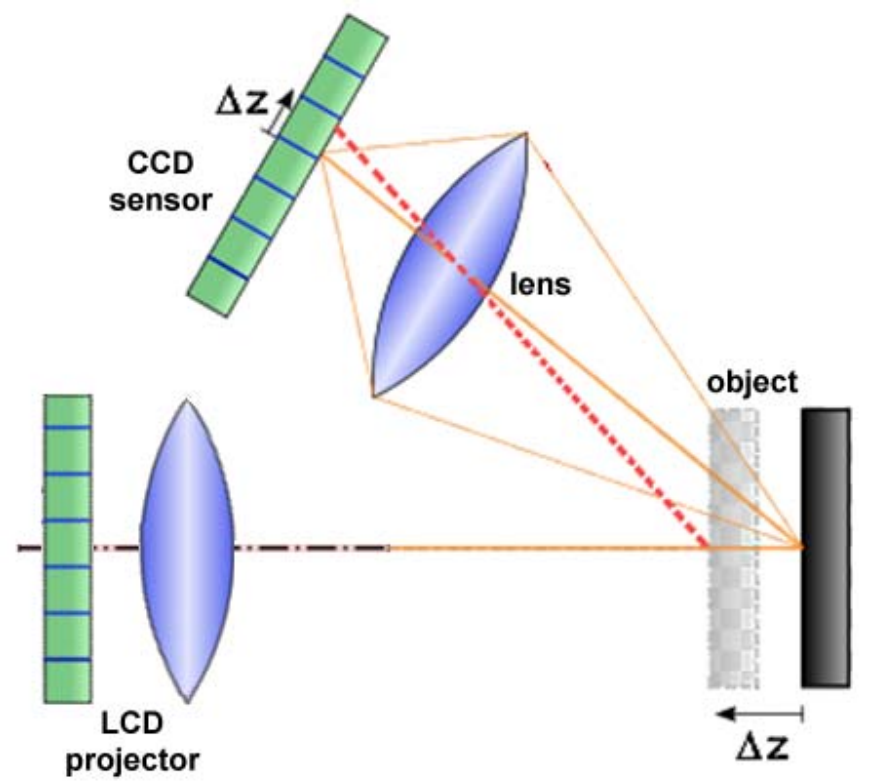

Fig. 3. Principle of triangulation by a couple $L C D$ projector/camera (CCD sensor)

The process of triangulation still consists in determining the location of a 3D point of the object by measuring angles to it from known points (the projection centres of the video camera and the video projector).

Depending on the distance at which each stripe projected by the video projector strikes the surface of the object from a reference point $(\Delta Z)$, the point of that stripe appears at different places in the camera's field of view $(\Delta z)$. The triangle is now formed by the target dot on the object, the optical centres of the camera and the projector. The length of one side of the triangle, the distance between the camera and the projector is known. The angle of the projector corner is also known. The angle of the camera corner can be determined by looking at the location of the target dot in the camera's field of view. This information gives the location of the target dot corner of the triangle. In most cases several stripes, instead of a single target dot, is projected over the object to speed up the acquisition process.

The quality of 3-dimensional data reconstruction highly depends on the accuracy of the determination of optical centres of both video camera and video projector.

The 3-dimensional resolution depends on the type of structured pattern projected (number of patterns projected), on the CCD resolution of the video camera and on the number of sensors. The time of digitization of a human subject takes from 500 milliseconds to 12 seconds, depending on the type of structured pattern projected (single or multiple patterns) and on the number of sensors used.

The average resolution achieved by white-light 3D body scanners available on the market is comprised between 10 and 30 points $/ \mathrm{cm}^{2}$.

\subsection{Types of structured light projection (single or multiple patterns)}

As said in the previous chapter, the resolution of 3-dimensional data captured by 3D scanners based on white light projection is related to the type of projection process. The projection of a single pattern (see example in figure 4) is the simplest process of structured light projection. Each stripe must be 
minimum 4-pixel wide to enable a correct identification by the video camera. Therefore the 3D data reconstruction is not pixel-wise (1 pixel of the video camera does NOT lead to one voxel of the 3D surface).

Another constraint of single-pattern projection is the risk of wrong numeration (identification) of stripes. If two stripes, once deformed on the body, are so close to each other that they merge at certain locations, or if stripe colour confuses with underwear or skin colours, the numeration of stripe becomes wrong and it creates a gap between two parts of the reconstructed 3-dimensional surfaces, or even worst, a big hole in the 3D dataset.

Furthermore, to increase the resolution of the system, it is recommended to increase the number of sensors but merging too many 3-dimensional surfaces leads to additional errors.

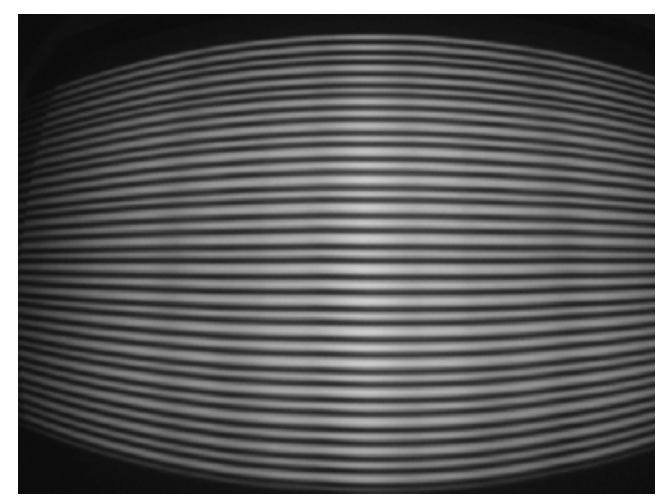

a.

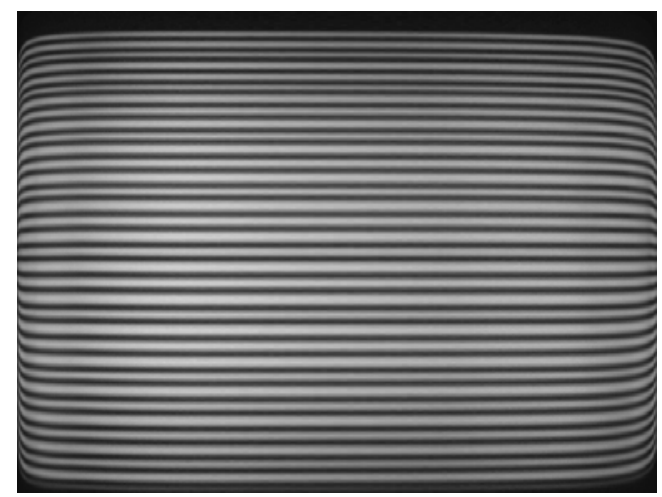

$b$.

Fig. 4. Example of single-pattern structured light

a. viewed by a video camera with wide-angle lens $-b$. after correction of geometric distortions

In order to avoid such problems, it is possible to project multiple patterns. Firstly, the use of an appropriate encoding of the structured light, known as Gray code (see figure 5), improves the numeration of stripes and therefore leads to a much more robust 3D reconstruction. Secondly, the projection of additional patterns, based on the Phase Shifting method (see figure 6) enables a pixelwise $3 \mathrm{D}$ reconstruction (1 pixel of the video camera LEADS to one voxel of the 3D surface) and therefore highly improves the resolution of the 3D dataset.

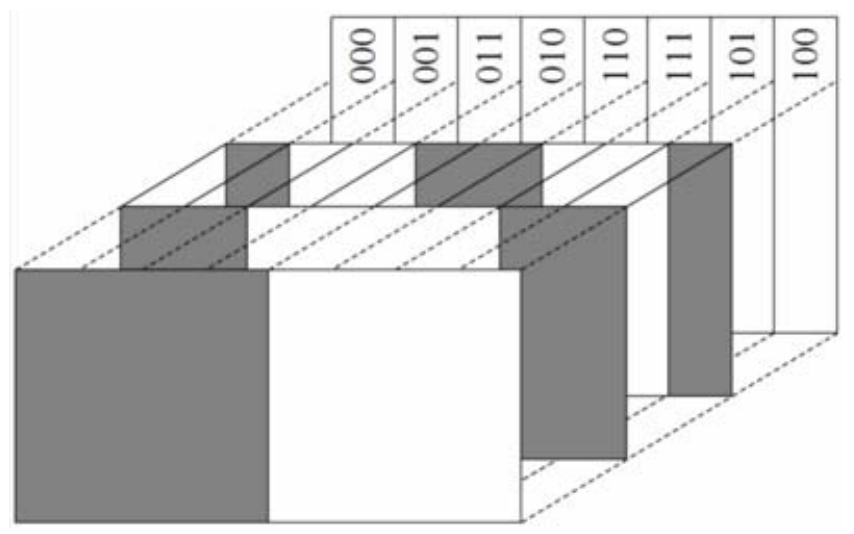

Fig. 5. Enhanced numeration of stripes through a special encoding of the structured light (Gray code). By projection of a sequence of binary coded stripe patterns, the subsequent light levels for each pixel are forming a unique binary word revealing the individual stripe number. To minimize the effect of bit errors, the stripe patterns are designed to change only one bit per stripe. 


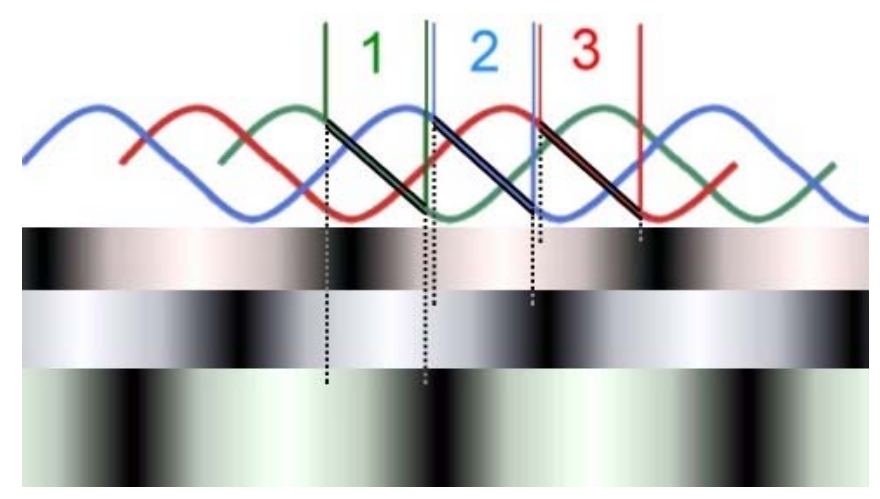

Fig. 6. Enhanced resolution through the projection of additional patterns (Phase Shifting method) Three phase-shifted patterns $(\pi / 2, \pi, 3 \pi / 2)$ are added to the reference pattern (full set of stripes) in order to enhance the resolution of the $3 D$ reconstructed dataset.

\subsection{D Body Scanning technologies based on stereo vision}

Stereo vision may also be used in the framework of 3D Body Scanning applications, provided the time of 3-dimensional surface reconstruction is not a key issue. It is notably used in Health and Medicine areas. Its principle is similar to human binocular vision. Two cameras (either digital cameras or high resolution video cameras) are used to obtain different views of the same subject. Common points are identified on both images. The exact 3D location of each such point is obtained by triangulation with the two optical centres of the cameras. The matching of both views may be facilitated by means of projection of a pattern (usually a randomized noise image). In that case, we speak of active stereo vision. Passive stereo vision (without pattern projection) may now be achieved by using high resolution digital cameras (> $10 \mathrm{Mpx})$.

These techniques are the most rapid in terms of time of 3D acquisition but the main problem is a very long time of 3D reconstruction (matching of both views and triangulation of all matched pixels) which reaches several minutes for each 3D surface, unless the resolution is drastically downsized.

\section{Comparison between the existing technologies for 3D Body Scanning}

\subsection{Laser scanning and triangulation}

The horizontal resolution of such body scanning devices depends on CCD resolution while the vertical resolution depends on the time of scanning.

The time of scanning of the whole body by laser scanners, based on vertical displacement of 3D sensors, is comprised between 12 and 24 seconds, for an average resolution comprised between 7 and 27 points per sq. $\mathrm{cm}$. Some laser scanners, based on rotating 3D sensors, may scan the whole body within 6 seconds but calibration and accuracy of such systems are less reliable. Such long times of scanning are acceptable when scanning static objects but they are not acceptable in terms of accuracy when scanning living subjects.

\subsection{Structured white light projection and triangulation}

The 3D resolution of such body scanning devices depends on CCD resolution but also on the number of patterns projected. The minimum width of each fringe of the projected pattern is 4 pixels. However a pixel-wise triangulation (each pixel of the CCD camera leads to one 3D voxel) may be obtained by applying the phase shifting method (see chapter 2.3 - figure 6). Furthermore a more reliable and robust 3D surface may be reconstructed through a specific encoding of projected fringed such as Gray code (see chapter 2.3 - figure 5). 
The time of digitizing of the whole body by structured white light projection devices is comprised between 0.6 and 12 seconds. Such systems can achieve an average resolution comprised between 10 and 30 points per sq. $\mathrm{cm}$. We can assess that the achieved accuracy is acceptable if the time of digitizing is lesser than 5 seconds.

\subsection{Impact of the amplitude of body sway on the accuracy of body measures}

Despite it is obvious that a too slow 3D scanning of a living subject will induce blur and inaccuracies, we hereby report how the amplitude of body sway was studied by specialists in biomechanics and then we analyze its impact on the accuracy of body measurements.

Several specialists in biomechanics experimentally studied the postural control of man standing at rest (David A. Winter et al., 1998) [1]. The evolution of the centre of pressure was recorded by an AMTI force platform for balance and sway analysis.

David A. Winter developed a 14-segment model to estimate the total body centre of mass (COM). It consisted of legs (2), thighs (2), lower arms (2), upper arms (2), pelvis and trunk (4). In order to measure the evolution of the centre of mass, David A. Winter tracked thanks to a 3D OPTOTRAK imaging system the movements of 21 infrared emitting diodes (IREDs) placed on the skin, from which it was possible to calculate at each moment the location of the centre of mass, taking into account the mass fraction of each subject's body segment.

Ten young adults (average age, $26 \mathrm{yr}$; body mass, $68.8 \pm 9.1 \mathrm{~kg}$, mean \pm SD) with no known balance or gait pathology were analyzed. Informed consent was obtained from each subject. Subjects were instructed to stand quietly on two force platforms with the feet in the side-by-side position at three different widths (ankle-to-ankle distance equal to 0.5, 1 and 1.5 times the distance between the hip joints, where the hip joint distance was estimated to be equal to the distance between the right and left anterior superior iliac spine). Subjects were instructed to stand quietly in each position for 2 min with eyes open. COP and COM was measured in a rigid manikin to estimate the net measurement noise in the force platforms and OPTOTRAK systems.

The centre of mass (COM) is estimated in three-dimensional space every $50 \mathrm{~ms}$. The superposition of the curves of the evolution of the centre of mass and of the centre of pressure (fig. 3) shows that the centre of pressure evolves on both sides of the centre of mass, as already shown by Schieppati et al. (1994) [2].

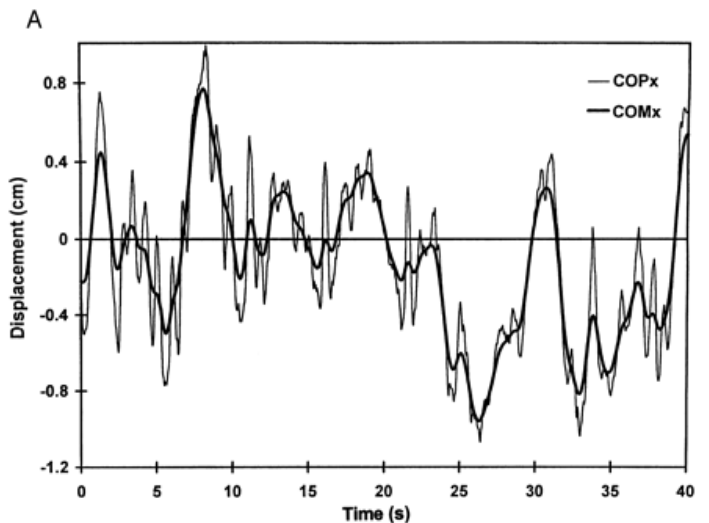

Fig. 7. From D.A. Winter et al, 1998

Compared displacements of the centre of mass (bold curve) and of the centre of pressure (thin curve) during a $40 \mathrm{sec}$. recording.

The amplitudes of centre of mass displacements recorded by David A. Winter on young adults vary between $\pm 0.8 \mathrm{~cm}$.

He has also shown that this sway amplitude is predicted to vary as a function of $K_{e}^{-0.5}$, where $K_{e}$ is the effective stiffness of the inverted pendulum. Therefore this amplitude will decrease when the subject increases the stiffness of his standing (such as the military standing to attention) which is not recommended for taking body measurements as such posture is not natural. However the sway amplitude will increase when the stiffness of the subject's standing decreases if he/she stands "at ease" or if the subject is a child or an elderly person $[3,4]$. 
By applying Euler's formula of approximation of the perimeter of an ellipse, we calculated the variation of a perimeter corresponding to the displacement of the subject's centre of mass. We gather in Table 8 a set of true body data (waist perimeters and their corresponding front and profile semi-axis values taken from 6 male subjects and 4 female subjects), the obtained variations of such waist perimeter are comprised between $2.4 \mathrm{~cm}$ and $3 \mathrm{~cm}$ (see table 8).

Table 8. Calculation of the waist perimeter variation with a body sway of $\pm 0.8 \mathrm{~mm}$

\begin{tabular}{|c|c|c|c|c|c|}
\hline Ref. & $\begin{array}{c}\text { Waist } \\
\text { girth }\end{array}$ & $\begin{array}{c}\text { Front } \\
\text { semi- } \\
\text { axis }\end{array}$ & $\begin{array}{c}\text { Profile } \\
\text { semi- } \\
\text { axis }\end{array}$ & $\begin{array}{c}\text { Body } \\
\text { sway }\end{array}$ & $\begin{array}{c}\text { Error } \\
\text { on } \\
\text { girth }\end{array}$ \\
\hline M1 & 86.1 & 16.8 & 13.3 & 0.8 & 2.4 \\
\hline M2 & 106.4 & 19.8 & 16.9 & 0.8 & 2.5 \\
\hline M3 & 89.9 & 18.5 & 14.2 & 0.8 & 2.5 \\
\hline M4 & 98.3 & 19.3 & 15.6 & 0.8 & 2.5 \\
\hline M5 & 112.8 & 20.3 & 17.9 & 0.8 & 2.5 \\
\hline M6 & 90.1 & 18.3 & 14.8 & 0.8 & 2.6 \\
\hline F1 & 64.7 & 15.1 & 10.9 & 0.8 & 2.7 \\
\hline F2 & 81.3 & 18.2 & 14.1 & 0.8 & 2.7 \\
\hline F3 & 72.7 & 17.2 & 12.0 & 0.8 & 2.6 \\
\hline F4 & 66.4 & 14.9 & 12.6 & 0.8 & 3.0 \\
\hline
\end{tabular}

As described above, this body sway value corresponds to the average amplitude of body sway movement measured at the centre of mass (at the waist area) of adult subjects. We recall that the sway amplitude will increase when the stiffness of the subject's standing decreases and notably if the subject is a child or an elderly person. Moreover, we can consider that the amplitude of the sway movement will increase at upper parts of the human body (at the chest area for instance).

By applying the same formula to a set of true body data (chest perimeters and their corresponding front and profile semi-axis values taken from the same 6 male subjects and 4 female subjects) but with a body sway of only $\pm 1.2 \mathrm{~cm}$, the obtained variations of such chest perimeter are comprised between $2.9 \mathrm{~cm}$ and $3.3 \mathrm{~cm}$ (see table 9).

Table 9. Calculation of the chest perimeter variation with a body sway of $\pm 1.2 \mathrm{~mm}$

\begin{tabular}{|c|c|c|c|c|c|}
\hline Ref. & $\begin{array}{c}\text { Chest } \\
\text { girth }\end{array}$ & $\begin{array}{c}\text { Front } \\
\text { semi- } \\
\text { axis }\end{array}$ & $\begin{array}{c}\text { Profile } \\
\text { semi- } \\
\text { axis }\end{array}$ & $\begin{array}{c}\text { Body } \\
\text { sway }\end{array}$ & $\begin{array}{c}\text { Error } \\
\text { on } \\
\text { girth }\end{array}$ \\
\hline M1 & 102.2 & 16.7 & 13.9 & 1.2 & 3.2 \\
\hline M2 & 110.7 & 17.9 & 14.9 & 1.2 & 3.2 \\
\hline M3 & 101.3 & 17.1 & 12.4 & 1.2 & 2.9 \\
\hline M4 & 111.5 & 17.1 & 14.7 & 1.2 & 3.1 \\
\hline M5 & 118.2 & 20.4 & 15.4 & 1.2 & 3.1 \\
\hline M6 & 102.4 & 17.7 & 12.7 & 1.2 & 2.9 \\
\hline F1 & 87.2 & 14.4 & 11.2 & 1.2 & 3.0 \\
\hline F2 & 101.6 & 17.6 & 13.2 & 1.2 & 3.1 \\
\hline F3 & 90.8 & 15.1 & 12.6 & 1.2 & 3.3 \\
\hline F4 & 92.2 & 14.2 & 12.9 & 1.2 & 3.3 \\
\hline
\end{tabular}

Therefore, the above analysis clearly shows that the crucial feature of a body scanner is its time of 3D data acquisition. It should be less than 5 seconds so that the body sway is less than $\pm 0.4 \mathrm{~cm}$ and therefore the perimeter variation is less than $1.2 \mathrm{~cm}( \pm 0.6 \mathrm{~cm})$. 


\section{SYMCAD ${ }^{\mathrm{TM}}$ II: a new generation of 3D body scanning technologies}

Having taken the measurements of over a million people inside institutions and clothed them, TELMAT released the SYMCAD ${ }^{\mathrm{TM}}$ II system, an unprecedented innovation in 3D body scanning and measurement technologies.

This new generation of 3D body scanners developed by TELMAT combines the projection of highly encoded structured light and optical triangulation between two high resolution cameras (stereo vision). Furthermore the projection of multiple patterns (Gray code and phase shifting) leads to a much better resolution than systems using single-pattern structured light projection.

The new SYMCAD ${ }^{\mathrm{TM}}$ II technology is ultra-fast and accurate. It takes less than a minute for the equipment to start up. The cycle includes an automatic scope calibration for measurement, totally free of any human intervention, with an acquisition time of 0.5 seconds for the entire human body and a $3 \mathrm{D}$ resolution higher than 25 points per sq. $\mathrm{cm}$., SYMCAD II is unequalled on the market.

The SYMCAD ${ }^{\mathrm{TM}}$ II technology already benefits of all advantages of multiple-pattern structured light projection techniques (see chapter 2.3). By processing the optical triangulation between two cameras rather than between one camera and one LCD projector (see figure 10 below), it increases even more the accuracy of the system because the optical characteristics of professional video cameras (lenses) are better than those of video projectors.

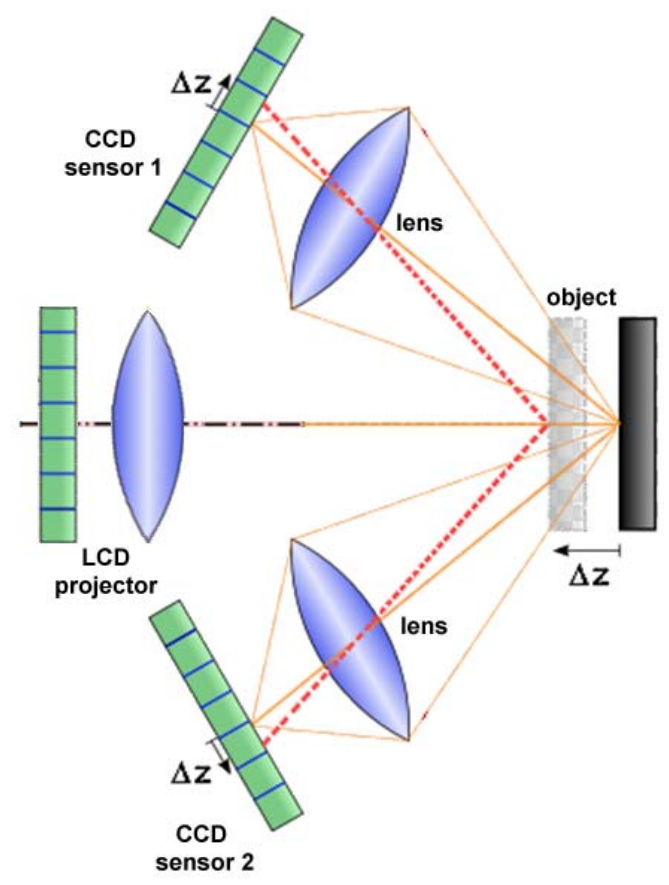

Fig. 10. Principle of optical triangulation by stereo vision SYMCAD ${ }^{\mathrm{TM}}$

\section{Perspectives: new possibilities for fashion and marketing and more ...}

Very easy to use, SYMCAD TM II provides boutiques and clothing store chains with an automated body measurement system that can determine clothing sizes and help produce made-to-measure clothes.

SYMCAD ${ }^{\mathrm{TM}}$ II helps provide made-to-measure comfort and elegance for the cost of ready-to-wear. SYMCAD ${ }^{\mathrm{TM}}$ II is a free-standing private booth and scanning system that is simple, autonomous and fun. Featuring automatic body positioning control, as well as a synthetic voice system that welcomes 
the customer into the booth, helps them find the ideal position, and then automatically triggers the measurement process. The person is inside a pleasant environment and a fun setting throughout.

Comfortable and fully preserving personal intimacy, SYMCAD ${ }^{\text {тM }}$ II features an exceptional spectrum of measurement, impervious to undergarment color or skin pigmentation. That is yet another SYMCAD ${ }^{\mathrm{TM}}$ II functionality unmatched on the market.

The realistic reconstruction of the human body and the software suite make it possible to collect any kind of special measurement. Measurements are automatically taken from characteristic body features. All measurements are based on raw data from the subject, free of any smoothing or data manipulation.

SYMCADTM II benefits are many. It obviously makes it possible to provide clothing that is perfectly adapted to the person $\downarrow$ s morphology, without alteration. Its automation promotes improved hygiene and privacy by limiting the fittings with personnel to the bare minimum. Shops can also thus manage a morphological database that will help optimize stocks and customer loyalty. Retail chains can enjoy an identical measurement tool in all their shops.

SYMCAD ${ }^{\mathrm{TM}}$ ST version (ST for Special Tracking) extends the range of applications of 3D body scanning to the domains of ergonomics, health and medicine. Specific scenarios of measurement extraction are defined to comply with all standardized predefined postures of the subject (sitting, standing or holding an object), according to the ISO-7250 standard. Once wireless active landmarks are set on the subject's body, SYMCAD ${ }^{\mathrm{TM}}$ ST automatically detects and identifies them and calculates the appropriate anthropometric measures.

Furthermore, SYMCAD ${ }^{\mathrm{TM}}$ enables to generate 3D avatars, either as 3D real data in VRML format, or 3D models warped from body measurement data for applications in virtual try-on or in entertainment.

\section{References}

1. Winter D. A., Patla A. E., Prince F., Ishac M., Gielo-Perczak K. (1998), Stiffness control of Balance in Quiet Standing, J. Neurophysiol., 80: 1211-21.

2. Schieppati M., Hugon M., Grasso M., Nardone A., Galante M. (1994), The limits of equilibrium in young and elderly normal subjects and in Parkinsonians, Electroenceph. Clin. Neurophysiol. 93, 286-298.

3. Gagey P-M., Bizzo G., Dimidjian J., Martinerie J., Ouaknine M., Rougier P. (1997), La danse du centre de pression et sa mesure - Posture et équilibre. Pathologies, vieillissement, stratégies, modélisation, (Ed M Lacour) (pp 167-180) Sauramps médical Montpellier

4. Gagey P-M., Toupet M. (1997), L'amplitude des oscillations posturales dans la bande de fréquence 0,2 hertz : étude chez le sujet normal - Posture et équilibre. Pathologies, vieillissement, stratégies, modélisation, (Ed M Lacour) (pp 155-166) Sauramps médical Montpellier 